\title{
MEAN VELOCITY OF THE ATLANTIC CURRENTS RUNNING NORTH OF SCOTLAND AND THROUGH THE ENGLISH CHANNEL
}

BY

\section{JOHAN GEHRKE}

W

hen the Atlantic current on its way towards north-east meets the continental shelf of the British Isles, it is divided into two branches, one of which goes through the Channel and passes into the North Sea, while the other and greatest branch continues its way northward along the Irish and Scottish west coast. It is the mean velocity of each of these two currents, that is to be calculated on the following pages. The calculations are based upon Martin Knudsen's hydrographical theorem ${ }^{1}$ ), by means of which it is possible from the fresh water supply to deduce the value of the water-volumes streaming in and out through a partially closed area of the sea, granting that the mean salinities of the in- and outgoing water-masses are known.

\section{Mean Velocity of the Water North of Scotland}

The Atlantic water-masses that stream northward along the west coasts of the British Isles, gradually get their salinity decreased, partly by direct rainfall, partly by fresh water supply directly from land and from the proportionally fresh water-volumes of the Irish Sea. In the areas north of the north-west of Scotland, the Atlantic current is divided into two branches, one of which turns southward into the North Sea, while the other turns in an easterly to north-easterly direction and enters the Norwegian Sea. We shall now calculate the mean velocity of the current at that place where these branches are just about to separate from each other; and we shall make the supposition that the velocity of the current has nearly the same value at

1) Martin Knudsen, Ein hydrographischer Lehrsatz. Annalen der Hydrographie und Maritimen Meteorologie. 1900. S. 316. 
all points of a section drawn from the north coast of Scotland in a north-westerly direction. (Comp. p. 14-15.)

We draw a plane vertical section $c$ out from Dunnet Head perpendicularly to the current-direction and stretching from surface to bottom; and from Landsend outwards we also draw a section $a$ from surface to bottom, but the shape of the section will be more

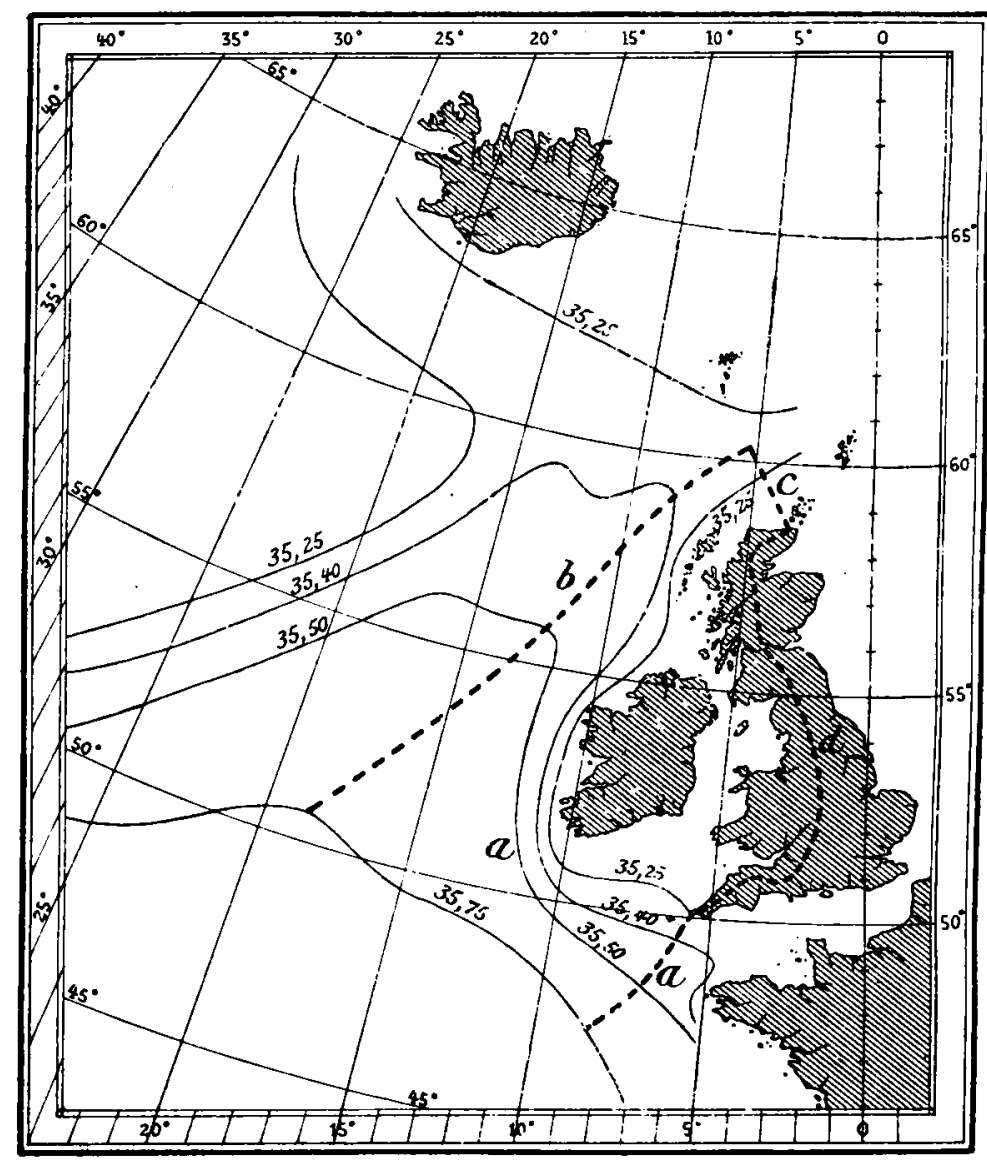

Fig. 1 NB. The unbroken curves are mean isohalines

exactly determined later on. The outer limits of $\boldsymbol{c}$ and $\boldsymbol{a}$ are joined by means of a section $\boldsymbol{b}$ (from surface to bottom), and the points where $\boldsymbol{c}$ and $\boldsymbol{a}$ reach the shores, are joined by a line $\boldsymbol{d}$ going through Scotland and England in such a manner as to form a division between the fresh waters running to the west coast and those running to the east and south coasts of the island. All the rainfall falling on the areas of Scotland and England west of the line $\boldsymbol{d}$ as well as the rain-fall of Ireland, with the exception of the water-masses that 
are again carried away by evaporation, will run into the sea-areas limited by the sections $\boldsymbol{a}, \boldsymbol{b}, \boldsymbol{c}$, and these sea-areas will also get a fresh-water supply from the rain-fall falling directly upon the seasurface.

Let the whole fresh-water supply to the areas in question during a unit of time be $\rho-\varepsilon$ (= rain-fall - evaporation). And let the salinity of the infinitely small element $d A$ of the limiting sections at the time $t$ be called $s$, and the normal component of the current at $d A$ be $i$ (considered as positive when going into the areas). Then according to the Hydrographical Theorem we have the equations:

$$
\left.\begin{array}{l}
\int_{a} d A \cdot \int_{0}^{T} i_{a} \cdot d t+\int_{b} d A \cdot \int_{0}^{T} i_{b} \cdot d t+\int_{c} d A \cdot \int_{0}^{T} i_{c} \cdot d t+(\rho-\varepsilon) \cdot T=0 \\
\int_{a} d A \cdot \int_{0}^{T} i_{a} s_{a} \cdot d t+\int_{b} d A \cdot \int_{0}^{T} i_{b} s_{b} \cdot d t+\int_{c} d A \cdot \int_{0}^{T} i_{c} s_{c} \cdot d t=0
\end{array}\right\}(\mathrm{I})
$$

$T$ indicating a very long space of time, and the integrations being extended throughout the sections indicated by the indices.

These equations can now be simplified in a very high degree. The mean value of the current-component normal to $d A$ and the mean salinity throughout a long space of time will be determined by the equations:

$$
i=\frac{1}{T} \cdot \int_{0}^{T} i \cdot d t \quad \text { and } \quad s=\frac{1}{T} \cdot \int_{0}^{T} s \cdot d t
$$

In the case of $i$ or $s$ (or both) having a constant value independent of $t$, we have exactly:

$$
\frac{1}{T} \cdot \int_{0}^{T} i s \cdot d t=\frac{1}{T} \cdot \int_{0}^{T} i \cdot d t \times \frac{1}{T} \cdot \int_{0}^{T} s \cdot d t=\ell \cdot s
$$

Besides the above mentioned case, this important equation will also have an exact validity in a large number of cases, when $i$ and $s$ varie periodically. Suppose, that $i$ and $s$ can be expressed by means of the equations:

$$
\left.\begin{array}{l}
i=i+i_{1} \cdot \sin \left(a_{1} t+\alpha_{1}\right)+\ldots+i_{n} \cdot \sin \left(a_{n} t+\alpha_{n}\right)=\boldsymbol{i}+\sum_{1}^{n} i_{p} \cdot \sin \left(a_{p} t+\alpha_{p}\right) \\
s=\boldsymbol{s}+s_{1} \cdot \sin \left(b_{1} t+\beta_{1}\right)+\ldots+s_{m} \cdot \sin \left(b_{m} t+\beta_{m}\right)=\boldsymbol{s}+\sum_{i}^{m} s_{q} \cdot \sin \left(b_{q} t+\beta_{q}\right)
\end{array}\right\}
$$

We then always can suppose

$$
a_{p}>0 \text { and } b_{q}>0
$$

and we get: 


$$
\begin{aligned}
\int_{0}^{t} i s \cdot d t= & i \boldsymbol{s} t-i \cdot \sum_{1}^{m} \frac{s_{q}}{b_{q}} \cdot \cos \left(b_{q} t+\beta_{q}\right)-\boldsymbol{s} \cdot \sum_{1}^{n} \frac{i_{p}}{a_{p}} \cos \left(a_{p} t+\alpha_{p}\right) \\
& -\sum_{1,1}^{n, m} \frac{i_{p} s_{q}}{2\left(a_{p}+b_{q}\right)} \cdot \sin \left[\left(a_{p}+b_{q}\right) \cdot t+\alpha_{p}+\beta_{q}\right] \\
& +\sum_{1,1}^{n, m} \frac{i_{p} s_{q}}{2\left(a_{p}-b_{q}\right)} \cdot \sin \left[\left(a_{p}-b_{q}\right) \cdot t+\alpha_{p}-\beta_{q}\right]+C
\end{aligned}
$$

where $C$ means a constant number independent of $t$.

This calculation is exact if we always have $a_{p}-b_{q}$ different from zero, that is to say, if all the terms in the series of $i$ have periods different from those of the terms in the series of $s$.

Since $\cos$ and $\sin$ never exceed the limits \pm 1 , all terms except the first in the last equation will disappear, when we divide the equation with $t$ and let $t$ grow infinitely. That is to say, we get:

$$
\frac{1}{T} \cdot \int_{0}^{T} i s \cdot d t=\frac{1}{T} \cdot \int_{0}^{T} i \cdot d t \times \frac{1}{T} \cdot \int_{0}^{T} s \cdot d t=i \boldsymbol{s}
$$

and (A) thus has an exact validity in this case.

However, the calculations above are not permissible, when $i$ and $s$ contain terms with equal periods, so that the case $a_{p}-b_{q}=0$ may occur. By an analogous proceeding we then get:

$$
\begin{gathered}
\int_{0}^{t} i s \cdot d t=\boldsymbol{i} \boldsymbol{s} t-\boldsymbol{i} \cdot \sum_{1}^{m} \frac{s_{q}}{b_{q}} \cos \left(b_{q} t+\beta_{q}\right)-\boldsymbol{s} \cdot \sum_{1}^{n} \frac{i_{p}}{a_{p}} \cos \left(a_{p} t+\alpha_{p}\right) \\
-\sum_{1,1}^{n, m} \frac{i_{p} s_{q}}{2\left(a_{p}+b_{q}\right)} \cdot \sin \left[\left(a_{p}+b_{q}\right) t+\alpha_{p}+\beta_{q}\right]+\sum \frac{i_{p} s_{q}}{2\left(a_{p}-b_{q}\right)} \cdot \sin \left[\left(a_{p}-b_{q}\right) t+\alpha_{p}-\beta_{q}\right] \\
+t \cdot \sum \frac{i_{p} s_{q}}{2} \cos \left(\alpha_{p}-\beta_{q}\right)+C .
\end{gathered}
$$

As to the two last sums, the first summing up shall be extended to all those combinations of $p$ and $q$ where $a_{p}-b_{p}$ is different from zero, and the second summing up shall contain all the combinations of $p$ and $q$ where $a_{p}-b_{q}=0$. By dividing with $t$ and extending the calculations over a very long space of time, we get:

$$
\frac{1}{T} \cdot \int_{0}^{T} i s \cdot d t=i s+\sum \frac{i_{p} s_{q}}{2} \cos \left(\alpha_{p}-\beta_{q}\right)
$$

Thus we see, that the equation (A) has generally no absolute validity in this case. Among the instances where the last term still disappears, we shall especially mention the case, where we always have: 


$$
a_{p}-\beta_{q}= \pm \frac{\pi}{2} \quad \text { when } \quad a_{p}-b_{q}=0
$$

Then all the terms of the sum will disappear, and we obtain again :

$$
\frac{1}{T} \cdot \int_{0}^{T} i s \cdot d t=i \mathrm{~s}=\frac{1}{T} \cdot \int_{0}^{T} i \cdot d t \times \frac{1}{T} \cdot \int_{0}^{T} s \cdot d t .
$$

The above calculations can always be carried out, no matter how great the number of terms in the series of $i$ and $s$ may be. The result must therefore still be true even if these numbers grow beyond any limit, that is to say, when $i$ and $s$ are expressed by means of infinitely prolonged convergent series.

The result of these investigations is: in the case of $i$ and $s$ varying periodically, the equation (A) keeps an exact validity, 1) if all the terms in the series of $i$ have periods different from those of the terms in the series of $s$; 2) if all such terms in the equations of $i$ and $s$ that have equal periods, also have a phase-difference of $1 / 4$ period. But if this last condition is not fulfilled, (A) will generally not be absolutely correct.

If we once more consider the plainest instances, we see that (A) is not correct in the case:

$$
\left.\begin{array}{l}
i=i+i_{1} \sin \frac{2 \pi t}{\tau} \\
s=\boldsymbol{s}+s_{1} \sin \frac{2 \pi t}{\tau}
\end{array}\right\}
$$

But (A) holds good in the cases:

$$
\left.\left.\begin{array}{rl}
i & =\boldsymbol{i}+i_{1} \sin \frac{2 \pi t}{\tau} \\
s & =\boldsymbol{s}+s_{1} \sin \frac{2 \pi t}{\theta}
\end{array}\right\}>\theta \quad \text { and } \quad \begin{array}{rl}
i & =\boldsymbol{i}+i_{1} \sin \frac{2 \pi t}{\tau} \\
\tau & =\boldsymbol{s}+s_{1} \cos \frac{2 \pi t}{\tau}
\end{array}\right\}
$$

Especially the last two equations are undoubtedly of considerable practical interest. The equations show that as long as the force $i$ of the current is superior to its mean value $i$, then the salinity will be constantly increasing or constantly decreasing; and as long as $i$ is inferior to the mean value $i$, then the salinity will be constantly decreasing, or constantly increasing. When the salinity attains its maximum or minimum, then the current will pass through the mean value, that is to say, then those forces will shift their direction which produce the variations of the current. This case gives a good picture of the tidal currents, and the phenomena of the ocean currents surely often have a similar character. 
We shall now pass on to a general exposition of the limits for the approximation attainable when (A) is used commonly in all cases. Let us suppose that the current $i$ is positive during the time from 0 to $t_{1}$, then negative during the period from $t_{1}$ to $t_{2}$, positive during the period from $t_{2}$ to $t_{3}$, and so on; should it happen that for a considerable period $i$ is zero, we shall then consider this period as a part of the preceeding period, that is to say: treat is as a period of positive current if the current was positive in the immediately preceeding period, but as a period of negative current in the opposite case. Further, let the greatest and the least salinity that upon the whole have occured in the positive current in the whole space of time from 0 to $T$ be

$$
s+s_{1} \text { and } \boldsymbol{s}+s_{2}
$$

and the greatest and least salinity in the negative current in the same time from 0 to $T$ be

$$
\boldsymbol{s}+s_{1}{ }^{\prime} \text { and } \boldsymbol{s}+s_{2}{ }^{\prime},
$$

$s_{1}, s_{2}, s_{1}{ }^{\prime}, s_{2}{ }^{\prime}$ being positive or negative numbers. If we then (for the sake of greater perspicuity) indicate negative currents by the sign $-i^{\prime}$ (so that in the following $i$ as well as $i^{\prime}$ are positive numbers), we get:

$$
\begin{aligned}
\int_{0}^{T} i s \cdot d t=\int_{0}^{t_{1}} i s \cdot d t & +\int_{t_{2}}^{t_{3}} i s \cdot d t+\int_{t_{4}}^{t_{5}} i s \cdot d t+\ldots \\
& -\left\{\int_{t_{1}}^{t_{2}} i^{\prime} s \cdot d t+\int_{t_{3}}^{t_{4}} i^{\prime} s \cdot d t+\int_{t_{s}}^{t_{6}} i^{\prime} s \cdot d t+\ldots\right\}
\end{aligned}
$$

consequently :

$$
\begin{aligned}
\int_{0}^{T} i s \cdot d t \leqq \int_{0}^{t_{1}} i & \cdot\left(\boldsymbol{s}+s_{1}\right) d t+\int_{t_{2}}^{t_{3}} i \cdot\left(\boldsymbol{s}+s_{1}\right) d t+\int_{t_{1}}^{t_{5}} i \cdot\left(\boldsymbol{s}+s_{1}\right) d t+\ldots \\
& -\left\{\int_{t_{1}}^{t_{3}} i^{\prime} \cdot\left(\boldsymbol{s}+{s^{\prime}}_{2}\right) d t+\int_{t_{3}}^{t_{4}} i^{\prime} \cdot\left(\boldsymbol{s}+s^{\prime}{ }_{2}\right) d t+\int_{t_{5}}^{t_{6}} i^{\prime} \cdot\left(\boldsymbol{s}+s_{2}^{\prime}\right) \cdot d t+\ldots\right\}
\end{aligned}
$$

or:

$$
\begin{gathered}
\int_{0}^{T} i s \cdot d t \leqq \boldsymbol{s} \cdot\left\{\int_{0}^{t_{1}} i \cdot d t-\int_{t_{1}}^{t_{2}} i^{\prime} \cdot d t+\ldots\right\}+s_{1} \cdot\left\{\int_{0}^{t_{1}} i \cdot d t+\int_{t_{3}}^{t_{3}} i \cdot d t+\int_{t_{4}}^{t_{5}} i \cdot d t+\ldots\right\} \\
-s_{2}{ }^{\prime} \cdot\left\{\int_{t_{1}}^{t_{2}} i^{\prime} \cdot d t+\int_{t_{3}}^{t_{4}} i^{\prime} \cdot d t+\int_{t_{3}}^{t_{4}} i^{\prime} \cdot d t+\ldots\right\}
\end{gathered}
$$

we shall now write: 


$$
\begin{aligned}
& \frac{\int_{0}^{t_{1}} i \cdot d t+\int_{t_{2}}^{t_{3}} i \cdot d t+\int_{t_{4}}^{t_{5}} i \cdot d t+\ldots}{\left(t_{1}-0\right)+\left(t_{3}-t_{2}\right)+\left(t_{5}-t_{4}\right)+\ldots}=i_{+} ; \frac{\int_{t_{1}}^{t_{3}} i^{\prime} \cdot d t+\int_{t_{3}}^{t_{4}} i^{\prime} \cdot d t+\int_{t_{5}}^{t_{6}} i^{\prime} \cdot d t+\ldots}{\left(t_{2}-t_{1}\right)+\left(t_{4}-t_{3}\right)+\left(t_{6}-t_{5}\right)+\ldots}=i_{-}^{\prime} ; \\
& \left(t_{1}-0\right)+\left(t_{3}-t_{2}\right)+\left(t_{5}-t_{4}\right)+\ldots=p ; \quad \frac{\left(t_{2}-t_{1}\right)+\left(t_{4}-t_{3}\right)+\left(t_{6}-t_{5}\right)+. .}{T}=1-p .
\end{aligned}
$$

Then $i_{+}$indicates the mean value of the current-force in positive current, $i_{-}^{\prime}$ the mean value of the current-force in negative current; $p$ is the probability of having positive current at a given moment, $1-p$ the probability of having negative current at a given moment. $p$ and $1-p$ are numbers below the unit. - We now get:

$$
\frac{1}{T} \cdot \int_{0}^{T} i s \cdot d t \leqq \boldsymbol{t} \cdot \boldsymbol{s}+p i_{+} s_{1}-(1-p) i_{-}^{\prime} \cdot s_{2}{ }_{2}
$$

And by analogous reasonings we get:

$$
\frac{1}{T} \cdot \int_{0}^{T} i s \cdot d t \geqq \boldsymbol{i} \cdot \boldsymbol{s}+p i_{+} s_{2}-(1-p) i_{-}^{\prime} \cdot s_{1}^{\prime}
$$

It is to be noticed that all the forces of currents in these calculations are the components of the really existing currents normal to the section-element $d A$. In all the cases of the current running parallel to $d A$, the products $i s \cdot d t$ will disappear from the equation for $\int_{0}^{T} i s d t$; and by the determination of the numbers $s_{1}, s_{2}, s_{1}^{\prime}, s_{2}^{\prime}$ we may neglect all the cases, where $i=0$. Therefore, if observations of the current-directions are at hand together with the observations of salinity, or if it by any other way is possible to point out a relation between these things, we ought - before the limits of the variability of the salinity are determined - to neglect all such salinities that occur together with currents parallel to $d A$. In many cases this proceeding will considerably lower the scope of the salinityoscillations in question.

If we now - taking due notice of the above mentioned precaution - find that $\boldsymbol{s}_{0}$ is the numerically greatest deviation that any salinity reaches from the mean value $\boldsymbol{s}$, so that all salinities, as well in positive as in negative currents, lie between the limits $\boldsymbol{s}+\boldsymbol{s}_{0}$ and $\boldsymbol{s}-\boldsymbol{s}_{0}$, it is seen that:

$$
\begin{aligned}
& s_{1} \leqq \boldsymbol{s}_{0} \quad s^{\prime} \leqq \quad \boldsymbol{s}_{0} \quad s_{2} \geqq-\boldsymbol{s}_{0} \quad s^{\prime}{ }_{2} \geqq-\boldsymbol{s}_{0} \\
& s_{1} \leqq \boldsymbol{s}_{0} \quad-s^{\prime}{ }_{1} \geqq-\boldsymbol{s}_{0} \quad s_{2} \geqq-\boldsymbol{s}_{0}-s^{\prime}{ }_{2} \leqq \quad \boldsymbol{s}_{0} .
\end{aligned}
$$
limits :

If this be put into the two inequalities stated above, we get the

$$
\boldsymbol{i} \cdot \boldsymbol{s}+\left\{p i_{+}+(1-p) i_{-}^{\prime}\right\} \cdot \boldsymbol{s}_{0} \geqq \frac{1}{T} \int_{0}^{T} i s \cdot d t \geqq i \cdot \boldsymbol{s}-\left\{p i_{+}+(1-p) i_{-}^{\prime}\right\} \cdot \boldsymbol{s}_{0}
$$


and at the same time we have:

$$
i=p i_{+}-(1-p) i_{-}^{\prime} .
$$

If $i=0$, we get:

$$
p i_{+}=(1-p) i_{-}
$$

and then (B) can be written in the following manner:

$$
2 p i_{+} \cdot \boldsymbol{s}_{0} \geqq \frac{1}{T} \cdot \int_{0}^{T} i s \cdot d t \geqq-2 p i_{+} \cdot \boldsymbol{s}_{0}
$$

For the waters treated here, $\boldsymbol{s}_{\mathbf{0}}$ is quite negligible in proportion to $s$, and thus (B) makes it evident that the equation (A) can be used with great exactitude in all cases where $i$ is different from zero. And (C) shows that (A) can be also employed in the case of $i$ being equal to zero, only by neglecting small quantities of quite the same order as when $i$ is $>0$.

Thus we only commit a very little errorin using the equation

$$
\frac{1}{T} \cdot \int_{0}^{T} i s \cdot d t=\frac{1}{T} \cdot \int_{0}^{T} i \cdot d t \times \frac{1}{T} \cdot \int_{0}^{T} s \cdot d t=i \cdot s
$$

commonly in all cases.

As already mentioned, $\boldsymbol{s}_{0}$ indicates the greatest deviation that any salinity reaches from the mean salinity, granted that, if possible, all salinities occuring together with currents parallel to $d A$ are neglected.

Applying (A) in the equations (I), we now get:

$$
\left.\begin{array}{l}
\int_{a} \boldsymbol{i}_{a} \cdot d A+\int_{b} \boldsymbol{i}_{b} \cdot d A+\int_{c} \boldsymbol{i}_{c} \cdot d A+(\rho-\varepsilon)=0 \\
\int_{a} \boldsymbol{i}_{a} \boldsymbol{s}_{a} \cdot d A+\int_{b} \boldsymbol{i}_{b} \boldsymbol{s}_{b} \cdot d A+\int_{c} \boldsymbol{i}_{c} \boldsymbol{s}_{c} \cdot d A=0
\end{array}\right\}
$$

When the section $b$ is drawn in a suitable way, these equations can be subject to a further simplification.

At each point of the sea there exists a mean direction of the current defined by the equation

$$
\frac{1}{T} \cdot \int_{0}^{T} i \cdot d t=0
$$

where $T$, as usual, is a very long time, and $i$ means the component of the current in an arbitrarily chosen direction perpendicular to the mean direction of the current. If we advance an infinitely small distance in this mean direction, we arrive at a new point with a new 
mean direction infinitely different from the former; and continuing in this manner, we produce a mean-current-curve. Through each point of the sea such a curve can be drawn, and for the areas considered here these curves can be regarded as running horizontally.

Now it is a well-known fact that the waters in the eastern part of the North Atlantic stream along the British continental shelf up towards the channel between Scotland and the Faroes, that is to say mainly parallel to the area of the greatest salinities on the chart Fig. 1; the mean isohalines of this chart are drawn by graphical adjustment of the various surface maps in the "Bulletins des Résultats etc." That the water-masses really must move parallel to the area mentioned, is also evident from the following reasoning: the waters of the Atlantic current may be considered as a water-volume streaming over and through fresher layers. The outward parts of the current must therefore first be subject to dilution, and the salinity will remain most unaltered in the heart of the current. The area of the maximum salinities will therefore indicate the current-direction, and the above-mentioned current-curves will run parallel to the area. - This reasoning will still hold true if the water-body splits into a number of separate branches, then the area of maximum salinity within each single branch must indicate the current-direction for this branch.

It may consequently be possible to lay through the area of the greatest salinities a curved surface stretching from surface to bottom (probably obliquely to the water surface), everywhere running parallel to the mean current-directions and having these lines as tangents. The intersection between this surface and the water surface is in Fig. 1 indicated by the curve $\boldsymbol{b}$.

For each element of this surface we have the equation:

$$
\frac{1}{T} \cdot \int_{0}^{T} i \cdot d t=0
$$

$i=$ the component of the current perpendicular to the element.

Consequently the water-volumes streaming through a quite arbitrarily chosen bit of this surface during a long space of time must also be equal to zero.

According to the equation (A) we must for each element of the surface mentioned also have the equation:

$$
\frac{1}{T} \cdot \int_{0}^{T} i s \cdot d t=0
$$

that is to say: The salt masses passing through a quite arbitrarily chosen bit of this surface during a long space of time will also be equal to zero. 
If we let a mean-current-curve move continually in a quite arbitrary way we produce a current-surface, for which the two equations (D) and (E) have validity as well as for the above mentioned maximum surface, yet with such an approximation which the equation (A) in each case can yield. (A) not always being quite exact, it is evident that (E) too can generally afford but an approximation to the truth, and for this very reason it is most advantageous to use just the chosen maximum surface. In the area of the greatest salinities we must expect to find the slightest salinity rariations; and on examining the various surface charts of the "Bulletins des Résultats etc." we get the impression that a great number of the salinity variations are due to water-motions parallel to the surface in question. As mentioned above, these variations shall be neglected when determining the scope of all the variations; and when we combine these circumstances with the very favourable conditions which the expressions $(B)$ and $(C)$ give us, we certainly shall ascertain that in the case of the maximum surface the equations (D) and (E) can be employed with an almost absolute exactitude.

If we now let the $\boldsymbol{b}$-section coincide with that part of the maximum surface lying between the sections $\boldsymbol{c}$ and $\boldsymbol{a}$, the equations (II) get the shape:

$$
\left.\begin{array}{l}
\int_{a} \boldsymbol{i}_{a} \cdot d A+\int_{c} \boldsymbol{i}_{c} \cdot d A+(\rho-\varepsilon)=0 \\
\int_{a} \boldsymbol{i}_{a} \boldsymbol{s}_{a} \cdot d A+\int_{c} \boldsymbol{i}_{c} \boldsymbol{s}_{c} \cdot d A=0
\end{array}\right\}
$$

We shall now suppose that the current has nearly the same force $I_{c}$ everywhere in section $c$ so that we have identically $i_{c}=I_{c}$. As $c$ is drawn perpendicularly to the direction of the current, then the normal current-component and the real current will in this case be equal. Further we let the section $\boldsymbol{a}$ coincide with the mean isohaline-surface for $35,50 \%, 00, \boldsymbol{s}_{a}$ thus becoming equal to $35,50 \%$ in all points of $\boldsymbol{a}$. Finally we shall for the sake of greater perspicuity introduce the indications:

$$
\begin{aligned}
& \int_{c} d A=C=\text { the total area of } c \\
& \int_{c} \boldsymbol{s}_{c} \cdot d A=S_{c} \cdot C
\end{aligned}
$$

$S_{c}$ then being the mean salinity throughout the whole section $c$.

The equations (III) will then be:

$$
\left.\begin{array}{rl}
\int_{a} i_{a} \cdot d A+I_{c} \cdot C+(\rho-\varepsilon) & =0 \\
35,50 \cdot \int_{a} i_{a} \cdot d A+I_{c} \cdot S_{c} \cdot C & =0
\end{array}\right\}
$$


It may be noticed that besides the above-mentioned isohalinesurface $\boldsymbol{a}$ also will include a little connecting surface up to Landsend. Only if the mean salinity of this connecting surface had also been equal to $35,50 \% / 00$, the equation

$$
\int_{a} \boldsymbol{i}_{a} \boldsymbol{s}_{a} \cdot d A=35,50 \cdot \int_{a} \boldsymbol{i}_{a} \cdot d A
$$

would have been quite correct. Yet the difference between the mean salinity of the connecting surface and $35,50^{\circ} / 00$ is such a little fraction of $35,50 \%$ that it is quite admissible to neglect this surface in our calculations. (When the mean salinity of the connecting surface is judged to be $35,40 \%$ the number $35,50-35,40=0,1 \%$ is only 0,3 per cent of 35,50). In order to make the error as little as possible, we have drawn the connecting surface in such a way that its mean salinity becomes as great as possible that is to say: the connecting surface is drawn perpendicular to the isohaline surfaces.

Of (IV) we get:

$$
\begin{aligned}
I_{c} \cdot\left(35,50-S_{c}\right) \cdot C & =35,50 \cdot(\rho-\varepsilon) \\
I_{c} & =\frac{35,50}{35,50-S_{c}} \cdot \frac{\rho-\varepsilon}{C}
\end{aligned}
$$

The sign of $I_{c}$ ought really to be - , in accordance with the fact that the current streams away from the area under consideration, but it is most convenient to omit the negative sign.

By planimeter measurements on Fig. 2 which as well as the following figures 3 and 4 are drawn on the basis of Martin KnUdsen's and Miss Kirstine Smith's mean salinity charts $^{1}$ ), is found:

$C=20,55$ square-kilom.; $S_{c}=35,152^{\circ}, 00 ; \quad\left(35,50-S_{c}\right) \cdot C=7,15$.

According to Deutsche Seewarte's chart of the Atlantic Ocean (Pl. 29 D) and Joseph Reger's map of the rain-fall over Europe ${ }^{2}$ ), the yearly mean value of the rain-fall over our area is judged to be $1500 \mathrm{~mm}$. In the wet climate the evaporation will certainly not exceed about $1 / 3$, and rain-fall minus evaporation is therefore calculated to be 1 meter per year. Finally our whole area is according to measurements on Fig. 1 calculated to be $6 \cdot 10^{5}$ square-kilometers; the curve $\boldsymbol{d}$ is drawn partly by means of JOSEPH REGER's chart, partly by means of ANDreE's Handatlas and PhiLIPs' Handy Volume Atlas of the World.

1) Martin Knudsex and Miss Kirstine Smith, The Salinity of the North Sea and Adjacent Waters etc. Conseil Permanent International pour l'Exploration de la Mer: Rapports et Procès-Verbaux Vol. VI. 1906, p. XXVI.

2) Deutsche Seewarte, Atlantischer Ozean, Ein Atlas. Hamburg 1902. Joseph Rzarr, Regenkarte von Europa. Petermanns Mitteilungen. 1903, p. 11. 
From these quantities we now get the final result:

$I_{c}=4,4$ miles in 24 hours $=9,4 \mathrm{~cm}$. per sec. $=0,18$ knots.

The whole water-volume that yearly passes in about a northeasterly direction between Scotland and the broken 35,33\% $\% 0$-isohaline drawn on Fig. 2 is thus about $\mathbf{6 1 0 0 0}$ cubic-kilometers, and as mentioned above its mean salinity is $35,15 \%$.

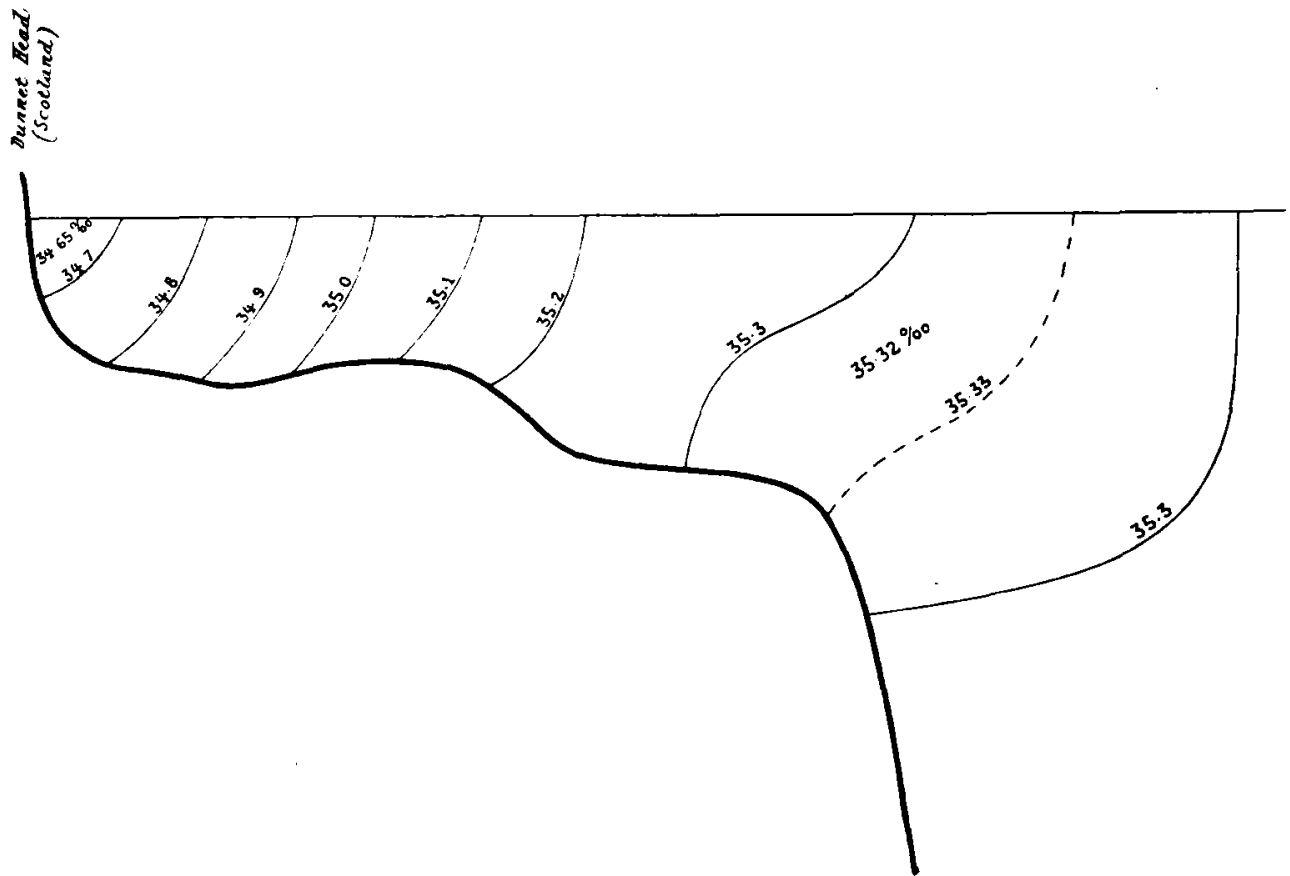

Fig. 2

Horizontal Scale: $1 \mathrm{~mm}$. rep. 2 Kilometers

Vertical Scale: $1 \mathrm{~mm}$. rep. 5 Meters

If we let the section $\boldsymbol{a}$ coincide with the $35,75 \%$-isohaline instead of with that of $35,50 \%$, the calculations can be carried out in quite the same manner, and we find:

$I_{\mathrm{c}}=4,3$ miles in 24 hours $=9,2 \mathrm{~cm}$. per sec. $=0,18$ knots.

These two results correspond very well with each other, and as the results might be considerably influenced by the most evident source of error in the preceeding calculations, viz. the supposition that the current has about the same force everywhere in section $c$, we may take the accordance between the results as a proof of the correctness of this assumption. 
We shall now let this source of error be subject to a closer examination, this being interesting in several respects. Let us first suppose, that $\boldsymbol{t}_{c}$ is exactly equal to $I_{c}$ everywhere in $c$. We then have quite correctly:

$$
\int_{c} i_{c} \boldsymbol{s}_{c} \cdot d A=I_{c} \cdot S_{c} \cdot C .
$$

If now, owing to some reason, the circumstances be altered so that the current $\mathbf{f}$. inst. decreases in the outer salter elements of $c$, and simultaneously increases in the inner fresher elements in such a way that the whole water-volume streaming through $c$ keeps quite the same value as before, then a smaller quantity of salt will now pass through $c$. In the transformation of the equations (III) into (IV) it then will be uncorrect to use the equation

$$
\int_{c} \boldsymbol{i}_{c} \boldsymbol{s}_{c} \cdot d A=I_{c} \cdot \int_{c} \boldsymbol{s}_{c} \cdot d A=I_{c} \cdot S_{c} \cdot C
$$

$\left(I_{c}=\frac{1}{C} \int_{c} i_{c} \cdot d A\right.$ now indicating the mean force of the current for the whole section $c$ ). We ought to use the calculation:

$$
\int_{c} i_{c} \boldsymbol{s}_{c} \cdot d A=I_{c} \cdot\left(S_{c}-d S_{c}\right) \cdot C \text {. }
$$

where $S_{c}-d S_{c}$ could be determined by giving those elements of $c$ where the current is stronger (in this instance the fresher elements) a greater weight than the other elements. Using $(G)$ instead of $(H)$, the elements with the strongest current generally have too little influence upon the calculations.

We thus see that a difference between the current-forces in the various points of $c$ generally alters the quantity of salt passing through $c$; and we must then take this circumstance into consideration by altering the value of $S_{c}$. In this case $S_{c}$ ought to be calculated by giving the single elements of $c$ weights proportional to the current-forces.

This circumstance will be of importance in using this method. The denominator of (V) includes the difference $35,50-S_{c}$; this is rather a small quantity, so that even small alterations of $S_{c}$ will considerably influence the value of $I_{c}$. Further such alterations will have different per-cent weights in the cases of the $35,50 \% 00$ - or the $35,75 \%$ isohaline-surface being chosen as $\boldsymbol{a}$-section. And the good accordance between the two results found above thus makes it evident, that the assumption of equal current everywhere in $c$ is sufficiently correct for our calculations. 


\section{The Current through the English Channel}

The Channel area may be limited by two vertical, plane sections Landsend-Porsal and Dover-Gap Gris Nez; and the two sections (Fig. 3 and 4) have been constructed by means of Martin Knudsen's and Miss Kirstine Smith's mean salinity maps.

On examining the various current charts in "The Pilots Handbook for the English Channel" ${ }^{1}$ ), especially plate 15, we see that the currents in Dover Strait may at any moment be considered as mainly parallel everywhere, but that the water-masses in the western entrance to the Channel form a rotation which during the various hours of the day runs sometimes in the same direction as the hands of a watch, sometimes in the opposite direction. From the relative position of the mean isohalines (compare the mean salinity charts cited above) to the current lines we may deduce that the northern-

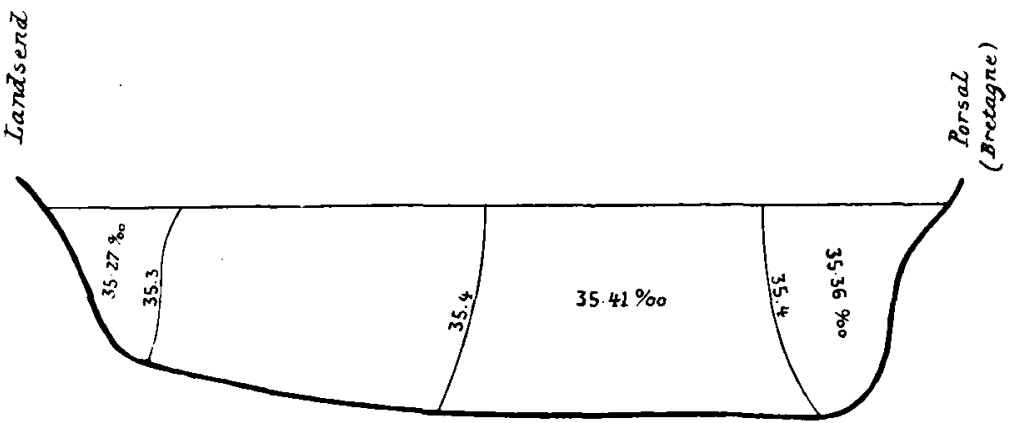

Fig. 3

Horizontal Scale: $1 \mathrm{~mm}$. rep. 2 Kilometers

Vertical Scale: $1 \mathrm{~mm}$. rep. 5 Meters

most $35,40 \%$-isohaline on Fig. 3 is to be regarded as the axis of the rotation.

The tongue of salt water stretching from Dover Strait into the North Sea shows that the North Sea receives a continuous salt water supply from the Channel which consequently must receive a salt water inflow from the Atlantic Ocean. The southern part of Fig. 3 having the greatest salinity, this Atlantic inflow mainly takes place through this area, which also accords with the fact that the currents are by the earth's rotation turned towards the right hand coasts.

We shall therefore calculate the mean currents for a long space of time in the Channel by assuming that a steady current having the force $x$ enters the North Sea through Dover Strait, and that the waters in the western entrance to the Channel form a steady rotation opposite to the watch-hands in such a way, that waters between the

1) Fourteenth Edition, London, 1902. 
northern 35,40\%-isohaline and the French coast enter the Channel with the uniform speed $\boldsymbol{e}$, while the waters between this isohaline and the English coast leave the Channel with the uniform speed $\boldsymbol{v}$. Indicating the two corresponding areas of Fig. 3 by $A_{e}$ and $A_{v}$, we find by means of the planimeter:

$$
\begin{gathered}
A_{e}=8,95 \\
\int_{v} s \cdot d A=316,7985=S_{e} \cdot A_{c} ; \quad S_{c}=3,94 \text { square-kilometers, } \\
\text { (It is to be noticed, that both the horizontal and the vertical seales } \\
\text { of this figure are the double of those of Fig. 3) } \\
\text { Horizontal Scale: } 1 \text { mm. rep. } 1 \text { Kilometer }
\end{gathered}
$$

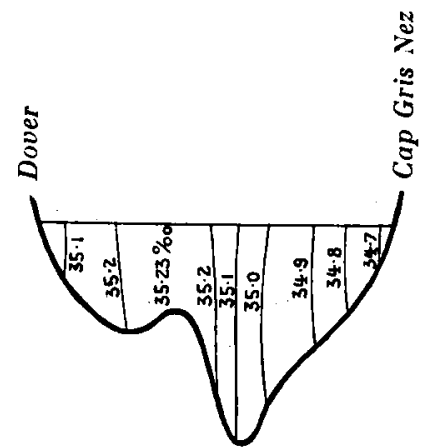

Thus the ingoing current has the mean salinity $35,40 \%$, while the salinity of the outgoing current is $\mathbf{3 5}, \mathbf{3 4} \%$. Besides that, we have:

The area of the section Dover-Cap Gris $\mathrm{Nez}=1,004$ square-kilometers, and the mean salinity is equal to $\mathbf{3 5 , 0 7 \%} \%$.

Further the fresh water supply to the Channel must be determined. By means of the previously cited rain-chart of Joseph REger and of ANDrEe's Handatlas, the area from which fresh water flows into the Channel is calculated to be 91785 square-kilometer's for the pertinent part of England and the Channel itself, and 122610 squarekilometers for the pertinent part of France. The yearly rain-fall evaporation is for the first mentioned area calculated to be $750 \mathrm{~mm}$. (REGER's chart), for the last area it is judged to be $450 \mathrm{~mm}$. Besides REGER's chart we have here employed as a basis that value of $\rho-\varepsilon$ for the drainage area of the Seine, that can be deduced from table VI in the essay of Sir John MurRay about the annual rain-fall ${ }^{1}$ ).

1) Sir John Murray, On the total annual rainfall etc. Scottish Geographical Magazine, February 1887. 
The total yearly rain-fall - evaporation $=$ fresh water supply to the Channel may thus be stated to be 124 cubic-kilometers.

The Hydrographical Theorem now gives us:

$$
\left.\begin{array}{l}
e \cdot 8,95+194=v \cdot 6,94+x \cdot 1,004 \\
e \cdot 8,95 \cdot 35,40=v \cdot 6,94 \cdot 35,34+x \cdot 1,004 \cdot 35,07
\end{array}\right\}
$$

As we have only two equations for the determination of three unknow quantities, we must try to get further information about the currents. In the already cited Pilots Handbook there is given on p. 149 some staternents from the lightship Varne from which it is possible to deduce, that the current in Dover Strait satisfies the equation

$$
i=\frac{1}{8}+\frac{17}{8} \cdot \cos \frac{2 \pi t}{12} \text { miles an hour }
$$

$t$ measured in hours and reckoned from the moment when the current at the "Varne" has its greatest velocity towards the North Sea. Currents towards the North Sea have a maximum speed of $21 / 4$ miles an hour and last for $6^{1 / 4}$ hours; currents towards the Channel have a maximum speed of 2 miles an hour and Jast for $5^{3 / 4}$ hours. - As

$$
\frac{1}{T} \cdot \int_{0}^{T} i \cdot d t=\frac{1}{8} \text { knots }=2027,9 \text { kilometers a year, }
$$

and as the current at the "Varne" can be taken as representing the currents everywhere in section 4 - at the surface as well as at the bottom -, we now get:

$$
\boldsymbol{x}=\mathbf{3} \text { miles in } 24 \text { hours }=6,4 \mathrm{~cm} \text {. per sec. }=1 / 8 \text { knots. }
$$

The whole annual water supply to the North Sea through Dover Strait is 2036 cubic-kilometers and its mean salinity is - as stated above - equal to $35,07 \%$.

When these numbers are filled into the equations (I), we find:

$\boldsymbol{e}=10,6$ miles in 24 hours $=22,7 \mathrm{~cm}$. per sec. $=0,44$ knots

$v=13,2$ miles in 24 hours $=28,3 \mathrm{~cm}$. per sec. $=0,55$ knots.

The three values for $\boldsymbol{e}, \boldsymbol{v}, \boldsymbol{x}$ give a representation of the watermovements in the Channel. 